\title{
Surgical Management of Bronchiectasis
}

\author{
Shanker Bhandari', Dinesh Gurung' Kazi Saiful Islam², Razzaque ${ }^{3}$, Md. Aftabuddin ${ }^{4}$, Asit Baran Adhikary \\ ${ }^{\prime}$ Resident (MS, CTVS), BSMMU, ${ }^{2}$ Asst. Professor, Thoracic Surgery, NIDCH, ${ }^{3}$ Professor, and HOD, Thoracic Surgery, NIDCH, ${ }^{4}$ Professor and \\ HOD, ${ }^{5}$ Professor, Cardiac surgery, BSMMU, Shahbag, Dhaka
}

\begin{abstract}
:
Background: Bronchiectasis is the permanent dilatation of the bronchi due to destruction of bronchial wall. Bronchiectasis still remains a serious problem in developing countries despite of modern medical facilities. Objectives: This study aims to demonstrate our surgical experience for bronchiectasis and analyze the risk factors related with the surgery outcome. Methods: Hospital based analyses of 39 patients were done who were underwent surgery for bronchiectasis and were on follow up in National Institute of Diseases of Chest hospital (NIDCH) in September 2014 to February 2015 were included in this study. All 39 patients had surgery for the bronchiectasis in the period of January 2014 to December 2014. Results: The mean age of the patients were 22.2 years. Twenty four patients were females and 15 were male. Symptoms were recurrent infection with cough, copious sputum in all patients and hemoptysis in 31 patients. The etiology was recurrent childhood infection in 17 patients, pneumonia in 11 patients, PTB in 6 patients, Aspiration in 2 patients, foreign body obstruction in 1 patient, and unknown etiology in 2 patients. Chest X-ray, CT scan and rigid bronchoscope were done for all patients. Bronchiectasis was left-sided in 17 patients. It was mainly confined to the lower lobes either alone in 9 patients and in conjunction with middle lobe or lingual in 8 patients. Indications for resection were failure of conservative therapy in 22 patients, hemoptysis in 8 patients, destroyed lung in 9 patients. Surgery was lobectomy in 24 patients, Bilobectomy in 5 patients, and pneumonectomy in 10 patients. Complications occurred in 10 patients with no operative mortality. Thirty four patients had relief of their preoperative symptoms after surgery in follow up periods. Conclusions: Surgical resection for bronchiectasis should be reserved for patients with localised disease who have failed medical management and have persistent symptoms that negatively affect their quality of life.
\end{abstract}

Keywords: Bronchiectasis, lobectomy, pneumonectomy.

[BSMMU J $2015 ; 8(2): 114-120]$

\section{Introduction:}

Bronchiectasis is an irreversible dilatation of bronchial tree resulting from destruction of the bronchial wall. Bronchiectasis was first described by Laenec in 1819 and, before the antibiotic era, it was considered a morbid disease with a high mortality rate from respiratory failure ${ }^{\prime}$. The clinical presentation varies and may involve repeated respiratory infections alternating with asymptomatic periods or with chronic production of sputum. Bronchiectasis should be suspected especially when there has been no exposure to tobacco smoke. The sputum

Corresponding Author: Prof. Md. Aftabuddin, Chairman Department of Cardiac Surgery, BSMMU, Telcphone: $+880-2-9661438$ Mobile: +880-1711-677713, Email: aftab12uddin@yahoo.com mixed with blood or there is recurrent hemoptysis ${ }^{2}$.

Today, with the improvement of health care and the availability of suitable antibiotics, the prevalence of bronchiectasis has declined and the patients with early disease can be treated successfully by conservative procedures in. Bronchiectasis still constitutes an important problem in developing countries because of tuberculosis, pneumonia, pertussis and serious rubella infections ${ }^{3}$. Current reports about the surgical management for bronchiectasis show that limited localized disease is associated with good postoperative outcome $e^{4}$.

This study was designed to analyze the cases of bronchi- 
actesis in our tertiary level hospital in regards to presentation, etiology, diagnostic aporoches, indications for surgery, surgical approach, and the outcome.

\section{Methods:}

We retrospectively reviewed the medical documents and took the perioperative history of 39 patients who underwent surgical resection for bronchiectasis between January 2014 to August 2014, at the Department of Thoracic Surgery in National institute of Chest Disease Hospital (NICDH). These 39 patients were in follow up in thoracic surgery OPD from September 2014 to February 2015. Variables of age, sex, symptoms, etiology of bronchiectasis involved, inductions and types of surgery, mortality, morbidity and the result of surgical therapy were analyzed.

Pre operative Chest radiography (CXR), computed tomography of the chest (CT) (Fig-1) and pulmonary function tests were reviewed to evaluate the type, severity, and distribution of bronchiectasis. All patients had intensive chest physiotherapy, sputum culture and sensitivity tests. Preoperative Chest physiotherapy was continued until the daily volume of the sputum decreased to $30 \mathrm{~mL}$ or less. Rigid bronchoscope was performed for all patients for the removal of secretion and determining foreign body or endobronchial lesions and identifying the other causes of haemoptysis. The bronchial aspirate was sent for microbiologic culture. Prophylactic antibiotics were given for 48 hours prior to surgery to prepare all patients undergoing surgery.

Operations were carried out under general anaesthesia with a double-lumen endotracheal tube to avoid contamination by secretions to opposite lung. Posterolateral thoracotomy was used for all patients. Surgical resection was performed on the basis of area of affection of pulmonary tissue assessed by preoperative Chest CT scan. If the disease was limited to one lobe, lobectomy was done and when the whole lung was affected, pneumonectomy was performed. In some cases lingulectomy was also required. During pulmonary resection, excessive bronchial dissection was avoided, and peribronchial tissues were preserved. The bronchial stump was closed by using a polypropylene suture in two layers. All resected specimens were sent for histo-pathological examination in order to confirm the diagnosis.

Retrospective recording of pre-surgical data included demographic information, medical comorbidities, and symptoms of presentation, side and location, rigid bronchoscopy data, spirometry data, indication for surgery. We also collected data about the extent of resection and the postoperative morbidity. History obtained from all 39 patients at follow up and review of documents, showed that all patients received intensive chest physiotherapy, antibiotics and analgesics in post operative periods.

\section{Results :}

A total of 39 patients were reviewed who were underwent surgical treatment for bronchiectasis in our department from January 2014 to August 2014. The mean age of these patients was 22.2years. Female to Male ratio was 24/39. All patients were symptomatic. The presenting symptom was recurrent infection with productive cough in all patients. Copious amount of sputum and recurrent hemoptysis was found 31 patients. The duration of symptoms ranged from one to 9 year (mean $\pm \mathrm{SD} 3.7 \pm 2.3$ years). The possible etiologies of bronchiectasis, symptoms, side and lobe affected were listed in Table 1.

Postero-anterior and lateral chest X-ray and CT scan were done for all patients, but the diagnosis of bronchiectasis was based mainly on the chest CT scan finding. It determines the type and extent of bronchiectasis, rigid bronchoscope was performed in all patients. The disease was on left side in 17 patients and on the right side in 12 patients. The disease was bilateral in 10 patients. Bronchiectasis involvement was predominantly in the lower lobes. The left lower lobe was affected in 9 patients and right lower lobe in 8 . (Table I).

The indications for pulmonary resection were failure of medical therapy in 21 patients, recurrent or massive 
hemoptysis in 10 patients, collapse, and consolidation in 8 patients (Table 3). Posterolateral thoracotomy and complete resection of all diseased segments were performed in all patients except in patients with bilateral discase. Seventeen patients had a lobectomy, 8 patients had a bilobectomy, 1 patients had lingulectomy, and 7 patients had a pneumonectomy (Table II).

Complications occurred in 10 patients and included postoperative bleeding (in 4 patients) that required exploration in 1 patient and atelectasis requiring bronchoscopic suction in 3 patients, a persistent air leak (more than 7 days) in 1 patient and this patient was proved to have bronchopleural fistula later on, post pneumonectomy space infection in 3 patients, wound infection in 1 patients, and post operative space collection in 1 patient.

Follow-up data were obtained for 39 of the patients. The mean follow-up of these patients was 4.5 months (range from 1 to 11 months). The symptoms disappeared in 32 patients and 5 patients had residual symptoms and controled by antibiotic. During the follow up period, 2 patients developed recurrent hemoptysis. These 2 patients were considered for further resection. Among 2 patients, 1 patient had left lower lobectomy and in follow up, this patient was planned for left side pneumonectomy. Right lower lobectomy was planned for another 1 patient for whom middle lobectomy was done because of frequent re-admission due to recurrent chest infection and hemoptysis after 5 months of follow up.

Table-I

Etiology, symptoms, side and lobe of Bronchiactesis.

\begin{tabular}{ll}
\hline variables & Numbers \\
\hline
\end{tabular}

Symptoms

$\begin{array}{lll}\text { Cough } & 39 & 100 \% \\ \text { Recurrent infection } & 39 & 100 \% \\ \text { Sputum } & 39 & 100 \%\end{array}$

Hemoptysis

Causes of bronchiectasis

Recurrent childhood

infection

$43.5 \%$

Pneumonia

TB

FB

$2.56 \%$

Unknown

$5.12 \%$

Side

Left 17

Right

Bilateral

Lobe affected

Left lower lobe 09

Right lower lobe

Middle lobe

$7.69 \%$

Left upper lobe

$2.56 \%$

Right upper lobe

$2.56 \%$

Right lower and middle lobes

$20.51 \%$

Lingual

$7.69 \%$

Left lower lobe and lingual

$5.12 \%$

Right upper lobe and middle

$5.12 \%$

Left pneumonectomy

$03 \quad 7.69 \%$

Right pneumonectomy

04

$10.2 \%$
Table -II

Indications, Types and complications of surgery

\begin{tabular}{lc}
\hline Variables & No of patients \\
\hline Surgery & \\
Lobectomy & 24 \\
Lower lobe & 15 \\
Upper lobe & 05 \\
Middle & 04 \\
Segmentectomy (Lingula) & 03 \\
Bilobectomy & 08
\end{tabular}


Left lower lobe and lingula

Right upper and middle lobes

Right lower and middle

Pneumonectomy

Complications

Post-operative hemorthage

Persistent air leak

01

Post pneumonectomy space infection

03

Broncho-pleural fistula

01

Wound Infection

01

Post op space collection (Effusion)

01

\section{Table-III}

Indication for surgery'

Indications of surgery

No, of Patients

Failure of medical treatment

21

Massive or recurrent hemoptysis

10

Destroyed lung

08

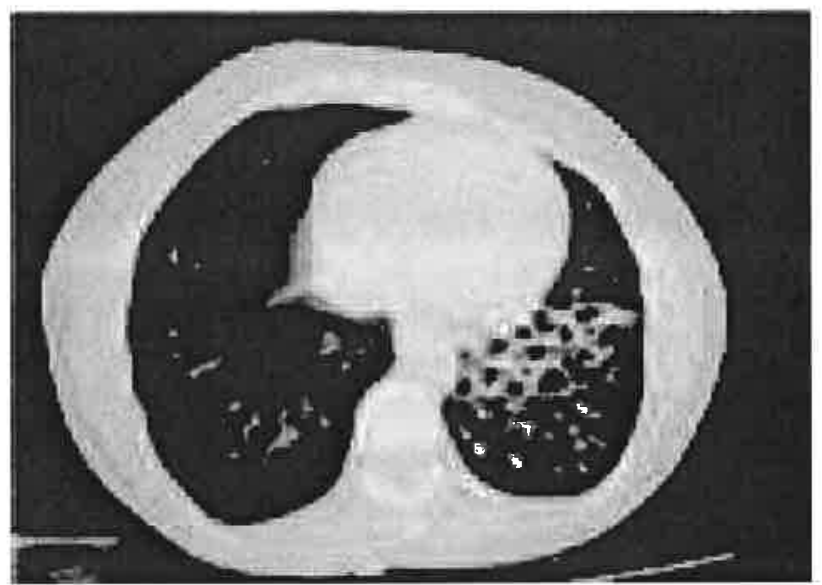

Fig-1: Chest CT scan showing bilateral cystic Bronchiectasis in the lower lobes.

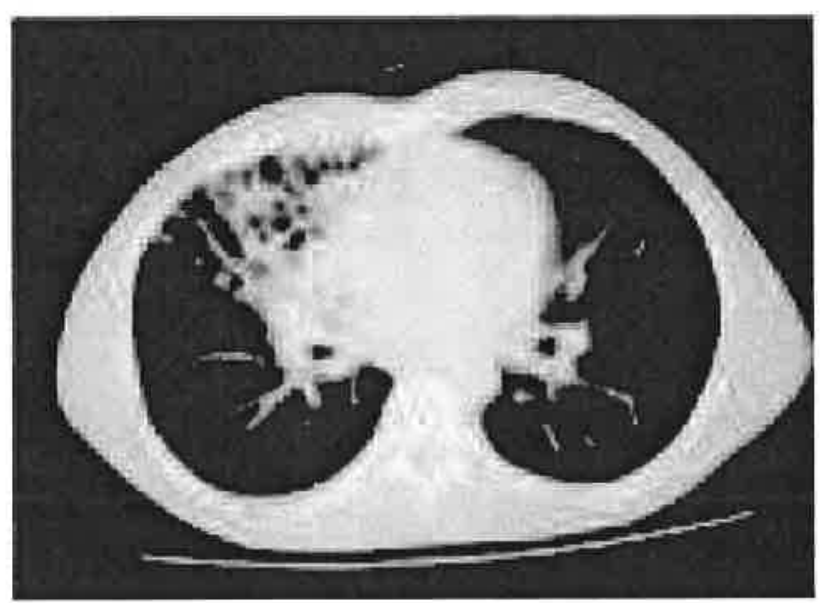

Fig-2: Chest CT scans showing the Bronchiectasis in the right middle lobe.

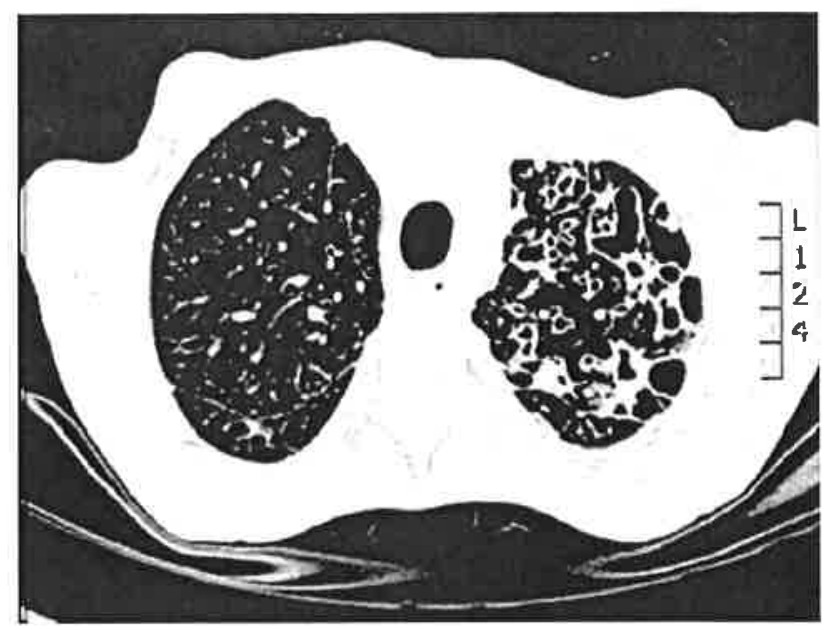

Fig-3: CT chest shows left upper lobe Bronchiectasis.

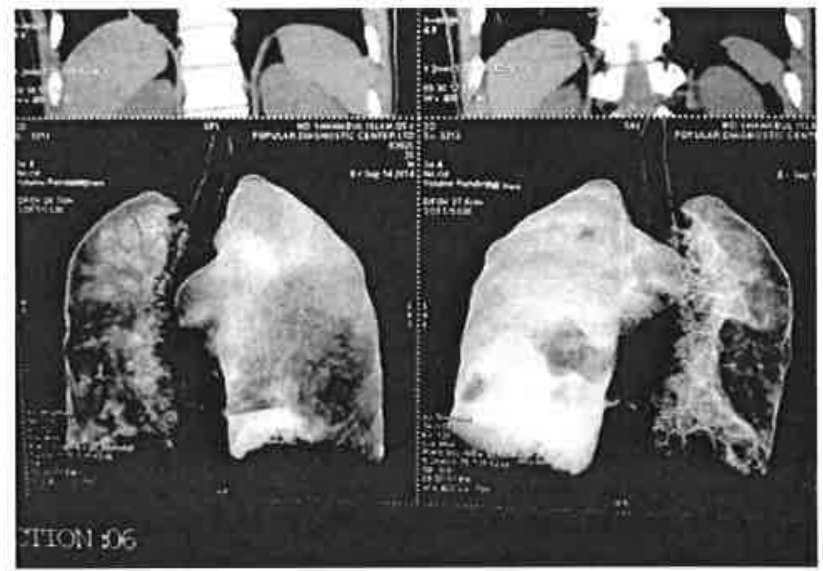

Fig-4: 3 D reconstruction CT scan of chest showingv Bronchiectasis in right lung.

\section{Discussion:}

Bronchiectasis is pathologically defined as a condition in which there are abnormal and permanent dilatations of proximal bronchi ${ }^{1,5}$. Before the antibiotic era the disease was considered a morbid one with a high mortality rate from respiratory failure ${ }^{3,5}$. It is usually caused by pulmonary infections or bronchial obstruction ${ }^{3,4}$. The incidence of bronchiectasis is unknown $n^{3,5}$. The prevalence of bronchiectasis decreased significantly over recent decades due to the proper use of antimicrobial therapy and immunization against viral and bacterial agents. Early 
recognition of foreign bodies with bronchoscopy also decreased the incidence of post-obstructive bronchiectasis $^{8}$. However, the incidence of bronchiectasis is still high in developing countries ${ }^{8.9}$. In spite of the advances in thoracic surgery, the optimal treatment for bronchiectasis remains controversial ${ }^{10}$.

Vendell and colleagues reported different causes of bronchiectasis including post infection, bronchial obstruction, immune deficiency, impaired mucociliary clearance, inflammatory pneumonitis, structural airways abnormalities and association with other disease ${ }^{2}$. They also reported that the cause remains unknown in a fairly high percentage of patients ranging from $26 \%$ to $53 \%{ }^{2,11}$. Recurrent pulmonary infection was most common cause in our 17 patients; pneumonia either in childhood or adulthood was the cause in our 11 patients, pulmonary tuberculosis in 6 patients and foreign body obstruction in 1 patient. Unknown etiology was in 2 patients (5.12\%) which is less in our study then other study. In our study, there was mixtured of etiologies. ${ }^{7,12}$

Patients with bronchiectasis typically present with recurrent respiratory tract infections, productive cough, bronchial suppuration and purulent bronchorrhea ${ }^{1,13}$. Cough, purulent and fetid sputum, and hemoptysis are the most common symptoms in other series ${ }^{10,13}$.

Diagnosis of bronchiectasis is based on clinical history and CT scan findings ${ }^{1,14}$. All the patients in our study had done CT scan of chest. CT criteria for diagnosis of bronchiectasis are well established (internal diameter of the bronchus more than 1.5 times than that of accompanying artery and evidence of lack of tapering of bronchi ${ }^{2,14}$. Bronchoscopy is not a main diagnostic method for bronchiectasis, but it may be helpful in identifying and removing foreign bodies, for locating the site of bleeding in patients with hemoptysis, and for diagnosing narrowed bronchi or neoplasms ${ }^{9.12}$. Preoperative bronchoscopy should be routinely done to rule out benign or malignant cause of obstruction ${ }^{3}$. In our study, rigid bronchoscopy was performed for all patients for the above mentioned reasons in addition to preoperative cleaning of the tracheobronchial tree in preparation of the selected patients for surgery.

In general, bronchiectasis affects most dependent portions of the lung, which includes posterior basal portions of the lower lobes, middle lobe and lingula. Overall one third of bronchiectasis is unilateral and affects a single lobe, one third is unilateral but affects more than one lobe, and one third is bilateral ${ }^{1,10}$. In our study, the disease affected the left lung in 17 patients. It was mainly confined to the lower lobes alone in 9 patients or in conjunction with middle lobe or lingual in 8 patients.

The initial treatment strategy for all patients with this disease should be conservative. Infection control, bronchodilatation and chest physiotherapy with postural drainage were the main components of conservative treatment. If medical treatment is unsuccessful or frequent episodes of hemoptysis exist surgical therapy should be considered ${ }^{1,3}$. As was the case with other series ${ }^{6,12}$, the indications for surgery in our study were failure of medical therapy, recurrent or massive hemoptysis, collapse with consolidation on radiological findings.

The goals of surgical therapy for bronchiectasis were to improve the quality of life and to resolve complications such as recurrent respiratory tract infection, severe or recuirent hemoptysis, and lung abscess. There was also consensus that, because bronchiectasis is a progressive disease, affected regions should be resected in a way that preserved uninvolved lung parenchyma, and early pulmo- 
nary resection is preferred if the diseases is localized 9.10 Complete and anatomical resection should be done with preservation of as much lung function as possible.

Therefore every type of resection is possible for these purposes. Ultimately, a minimum of two lobes or six pulmonary segments must be spared to ensure adequate pulmonary function ${ }^{1,14}$. For successful surgery, Kutly and colleagues recommend that the operation should be performed in 'dry period', complete resection of suspected areas by intraoperative examination that could not be determined by radiological examination to decrease relapse rates, and after lung resection in childhood lung can still grow to occupy the space ${ }^{10}$.

Apart from the 7 patients who underwent pneumonectomy for collapse with consolidation (destroyed lung), most of our patients had a localized disease to one or more lobe. Complete resection was achieved in 37 patients. Bilateral bronchiectasis a contraindication to surgical therapy in selected patients ${ }^{7,10}$. In our series, 10 patients had bilateral bronchiectasis. A staged thoracotomy for these patients was performed.

Complication occurrence is $9.4-24.6 \%$ in the current literature; our result was $25.64 \%$ therefore our result ( 10 patients) is within the reported incidence ${ }^{12,14}$. It included early and late complications. Early complications included bleeding requiring exploration, post space infection, and prolonged air leak more than 7 days and the late one included the recurrence of symptoms and extension into other lobes or segments. Apart from the patient developed bronchopleural fistula, all complications were successfully treated. That patient developed post pneumonectomy space infection with persistent air leak and was proved to have bronchopleural fistula, was re-admitted 8 months after surgery with persistent air in post punemo- nectomy space, recurrent pus collection, and chest infection. Mortality ranges from $0 \%$ to $8.3 \%$ in the literature and current mortality is less than $1 \%{ }^{10.12}$. There was no operative mortality in our study.

In our study, patients with complete resection of a localized bronchiectasis had better outcomes than those with incomplete resection. Regarding symptoms, the results of surgery can be considered satisfactory in our experience. More than $82 \%$ of our patients had relieved their preoperative symptoms. These results are similar to other serjes 10,12

The follow up time was short as we depend mainly on the outpatient department visits. Our center is a referral center that covers a wide area and most of the patients with improvement of symptoms lost to follow up as they are far from the center and their concept that they need no more follow up.

\section{Conclusion :}

In conclusion, surgical resection of bronchiectasis can be performed with acceptable morbidity and without mortality of any age for localized disease by proper selection and preparation of the patients.Complete resection of the involved sites are required for the optimum control of symptoms and better outcome.

\section{References :}

01. Balkanli K, Genç O, Dakak M, Gurkok S, Gozubuyuk A, caylak $\mathrm{H}$,et al. Surgical management of bronchiectasis: analysis and short-term results. Eur J Cardiothorac Surg 2003; 24:699-702.

02. Vendrell M, de Gracia J, Olveira C, Martínez MA, Girón R, Máiz L, et al. Diagnosis and treatment of bronchiectasis. Spanish Society of Pneumology and Thoracic Surgery. Arch Bronconeumol 2008;44:629-640.

03. Agasthian T, Deschamps C, Trastek VF, Allen MS, Pairolero PC. Surgical management of bronchiectasis. Ann Thorac Surg 1996; 62:976-8; 979-980. 
04. Ashour M, Al-Kattan K, Rafay MA,. Saja KF, Hajjar W, Rahman A. Current surgical therapy for bronchiectasis. World $J$ Surg 1999;23:1096-1104.

05. Seaton D. Bronchiectasis. In: Seaton A, Seaton D. eds. Crofton and Douglas's respiratory diseases. Volume I. 5th Ed. New Dellii: Oxford University Press, 2000:794-808.

06. Prieto D, Bemardo J, Matos MJ, EugeÂnio L, Antunes M. Surgery for bronchiectasis. Eur J Cardiothorac Surg 2001; 20:19$23,23-24$

07. Sehitogullari A, Bilici S, Sayir F, Ufuk Cobanoglu and Ali Kahraman. A long-term study assessing the factors influencing survival and morbidity in the surgical management of bronchiectasis. J Cardiothorac Surg 2011; 6:161-167

08. Campbell DN, Lilly JR. The changing spectrum of pulmonary operations in infants and children. J Thorac Cardiovasc Surg 1982; 83:680-685

09. Otgün I, Kamak I, Tanyel FC, Şenocak ME, Büyükpamukçu N. Surgical treatment of bronchiectasis in children. J Pediatr Surg 2004;39:1532-1536.
10. Kutlay H, Cangir AK, Enön S, Ekber S, Akal M, Nezı A,et al. Surgical treatment in bronchiectasis: analysis of 166 patients. Eur J Cardiothorac Surg 2002;21:634-637.

11. Pasteur MC, Helliwell SM, Houghton SJ,. Webb SC, Foweraker $\mathrm{JE}$, Coulden RA, et al. An investigation into causative factors in patients with bronchiectasis. Am J Respir Crit Care Med 2000; $162: 1277-1284$

12. Yuncu G, Ceylan KC, Sevinc S, Ahmet UA, Seyda OK, Goksel $\mathrm{KG}$, et al. Functional results of surgical treatment bronchiectasis in a developing country. Arch Bronconeumol 2006;42:183-188.

13. Deslauries J, Goulet S, Franc B. Surgical treatment of bronchiectasis and broncholithiasis. In: Franco LF, Putnam JB. eds. Advanced therapy in thoracic surgery. Hamilton, ON: Decker; 1998:300-9.

14. Giovannetti $\mathrm{R}$, Alifano $\mathrm{M}$, Stefani A, Legras A, Grigoroiu $\mathrm{M}$, Collet JY, et al. Surgical treatment of bronchiectasis: early and long-term results. Interact Cardiovasc Thorac Surg 2008;7:609612. 
